\title{
In Silico and in Vitro Evaluation of Real Time PCR Assay for Detection of Staphylococcus aureus
}

\author{
Al-Shaimaa Muhammed Saeed AL-Rawi ${ }^{1}$, Abdul Kareem A. AlKazaz ${ }^{1}$, Majeed Arsheed Sabbah ${ }^{2}$ \\ and Mohammed Mahdi Al-Zubaidi ${ }^{2}$ \\ Department of Biotechnology, College of Sciences, University of Baghdad ${ }^{1}$, Forensic DNA Center, \\ Alnahrain University ${ }^{2}$, Baghdad-Iraq.
}

\begin{abstract}
Many research groups developed real time PCR assays for identification of Methicillin-resistant Staphylococcus aureus (MRSA). They designed different primers and probes in their assays. Gene mec $\mathrm{A}$ is the target identification of MRSA by PCR assay. The aim of this study is using in silico approach to identify the best primers and probe for real time PCR identification mecA gene. Published primers and probes were analyzed in silico to select the best for real time PCR identification of $m e c \mathrm{~A}$ gene. The selected primers and probe successfully used for real time amplification of twenty MRSA tested. This study reveals the importance of in silico approach for designing diagnostic assays shorten the cost and the time. [DOI: 10.22401/JNUS.20.1.18]
\end{abstract}

Keywords: Methicillin-resistant Staphylococcus aureus (MRSA), real time PCR, in silico.

\section{Introduction}

Methicillin-resistance staphylococcus aureus (MRSA) is a serious threat capable of causing different diseases worldwide [1]. Traditional detection of staphylococcus aureus isolates performed by using bacteriological and biochemical methods. While identification of methicillin resistance done by disk diffusion assay [2]. Modern methods used polymerase chain reaction (PCR) techniques for rapid identification of MRSA. Many genes used for specific detection staphylococcus aureus such as femA [3] and nuc [4] genes. The mec A gene used for detection of methicillin resistance [3]. Many researchers designed primers and probes for detection MRSA by real time PCR $[5,6,7]$. In silico approach was used in recent study to select the best primers and probe [8]. In this study in silico approach used to select the best primers and probe for detection mecA gene in local MRSA isolates using real time PCR assay.

\section{Materials and Methods \\ Bacterial isolates}

Total of 20 clinical isolates primary were identified as Staphylococcus species obtained from hospitalized patients at Child Central Hospital in Baghdad city. The isolates were primary cultivated on Mannitol agar medium then incubated at $37^{\circ} \mathrm{C}$ for $18-24 \mathrm{~h}$ in aerobic condition.

\section{DNA Extraction}

Boiling method was used for bacterial DNA extraction as described by [9] with some modifications. Extracted DNA subjected to $0.8 \%$ agarose gel electrophoresis then stained with Ethidium bromide. Purity and concentration determined by Nanodrop Spectrophotometer [7].

\section{Identification of MRSA by conventional Duplex PCR}

Two genes were selected to be amplified together in a duplex PCR technique (mec A and femA). PCR was performed to amplify femA gene as a specific genomic marker for $S$. aureus. Forward primer was (5'CATGATGGCGAGATTACAGGT-3') and Reverse primer was (5'GTCATCACGACCAGCGAAAGC-3') [8]. The length of PCR product is $314 \mathrm{bp}$. PCR amplification was performed for 35 cycles with initial denaturation $\left(95^{\circ} \mathrm{C} / 5 \mathrm{~min}\right)$, denaturation $\left(94^{\circ} \mathrm{C} / 1 \mathrm{~min}\right)$, annealing $\left(60^{\circ} \mathrm{C} /\right.$ $30 \mathrm{sec})$, extension $\left(72{ }^{\circ} \mathrm{C} / 45 \mathrm{sec}\right)$ and final extension $\left(72^{\circ} \mathrm{C} / 5 \mathrm{~min}\right)$ using Accupower master mix kit (Bioneer/ Korea).

The gene mecA was amplified because it encodes methicillin resistance. Forward primer was (5'-AAAATCGATGGTAAAGGTTGGC3') and Reverse primer was (5'AGTTCTGCAGTACCGGATTTGC-3') (8). The length of PCR product is 533bp. PCR 
amplification was performed for 35 cycles with initial denaturation $\left(95^{\circ} \mathrm{C} / 5 \mathrm{~min}\right)$, denaturation $\left(94^{\circ} \mathrm{C} / 1 \mathrm{~min}\right)$, annealing $\left(60^{\circ} \mathrm{C} /\right.$ $30 \mathrm{sec})$, extension $\left(72^{\circ} \mathrm{C} / 45 \mathrm{sec}\right)$ and final extension $\left(72^{\circ} \mathrm{C} / 5 \mathrm{~min}\right)$ using Accupower master mix kit (Bioneer/ Korea).

\section{In-silico real time PCR identification of MRSA}

Twenty five primers and probes published by different researches online collected and analyzed by the in silico PCR online software (http://insilico.ehu.es/PCR/) to select the primers that in silico amplify most Staphylococcus aureus strains. The selected primers and collected probes analyzed by oligoanalyzer 3.1.

https://eu.idtdna.com/analyzer/Applications/O1 igoAnalyzer/ to select the best forward and reverse prime and probe for Taqman real time PCR assay detection of mecA gene. The best in silico primes and probe analyzed finally by Blast (http://blast.ncbi.nlm.nih.gov) to identify the specificity for Staphylococcus aureus.

\section{Real time PCR for mecA gene}

The selected forward primer was (5'- GAATGCAGAAAGACCAAAGCA-3') and reverse primer was (5'- TTTGGAACGATGCCTATCTCA-3') and probe was (FAM-5' ACCGAAACAATGTGGAATTGGCCA-3'BHQ). The PCR product was $124 \mathrm{bp}$. Real time PCR conditions were initial denaturation $\left(95^{\circ} \mathrm{C} / 3 \mathrm{~min}\right), \quad$ denaturation $\left(94^{\circ} \mathrm{C} / 15 \mathrm{sec}\right)$, annealing $\left(60^{\circ} \mathrm{C} / 30 \mathrm{sec}\right)$, extension $\left(72^{\circ} \mathrm{C} /\right.$ $45 \mathrm{sec})$ and final extension $\left(72^{\circ} \mathrm{C} / 5 \mathrm{~min}\right)$ for cycles using Accupower master mix kit (Bioneer/ Korea).

\section{Results and Discussion}

The identity of Staphylococcus isolates were verified as $S$. aureus by cultivation on mannitol salt agar which considered selective and differential medium for the genus Staphylococcus [10]. The isolates had the ability to ferment mannitol sugar and form large, round, smooth, raised, mucoid and glistening. More over only S. aureus forms large golden colonies surrounded by wide yellow zones due to fermenting the mannitol and producing acid which turned the color of the medium from pink to Fig.(1). Antibiotic disk assay was used to confirm the methicillin sensitivity of isolates Fig.(2).

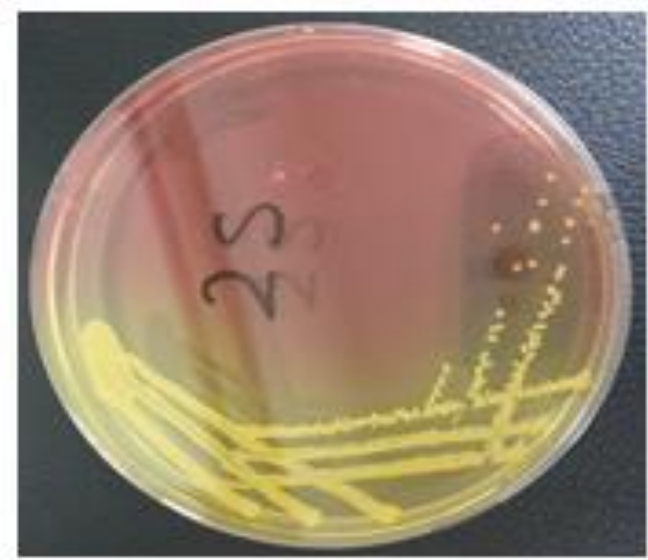

Fig.(1): Staphylococcus aureus colonies cultures on Mannitol.

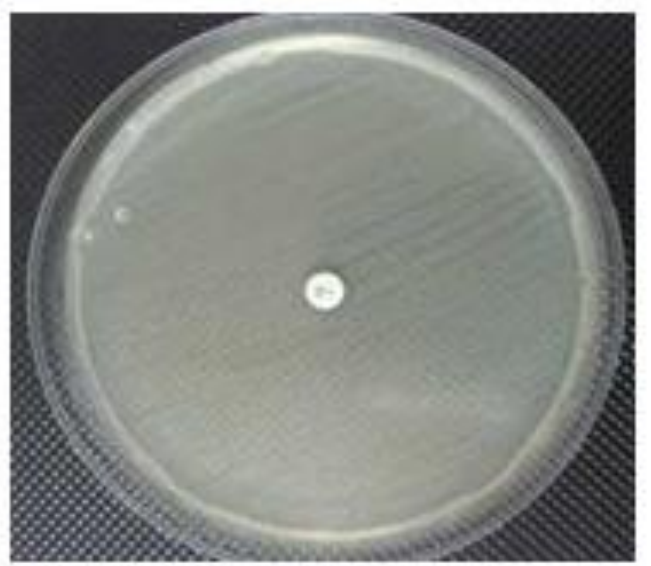

Fig.(2) Methicillin (5mg) resistance of $S$. aureus on Muller-Hinton agar.

To confirm the identity of MRSA isolates, DNA extracted from bacterial isolates Fig (3), and then Duplex PCR was used for amplification mecA and femA genes [3]. The gene mecA did not amplified in methicillin sensitive $S$. aureus isolates Fig (4).

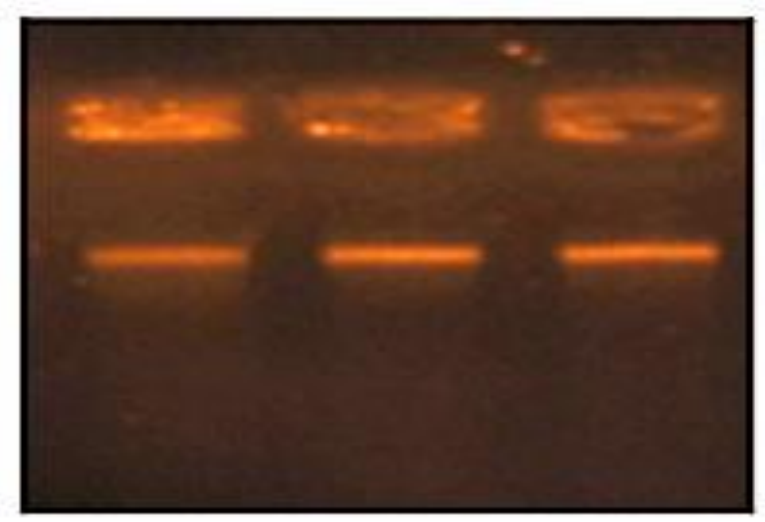

Fig.(3): Agarose gel electrophoresis $0.8 \%$ of DNA extracted from Staphylococcal isolates stained with ethidium bromide. 


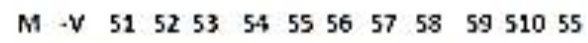

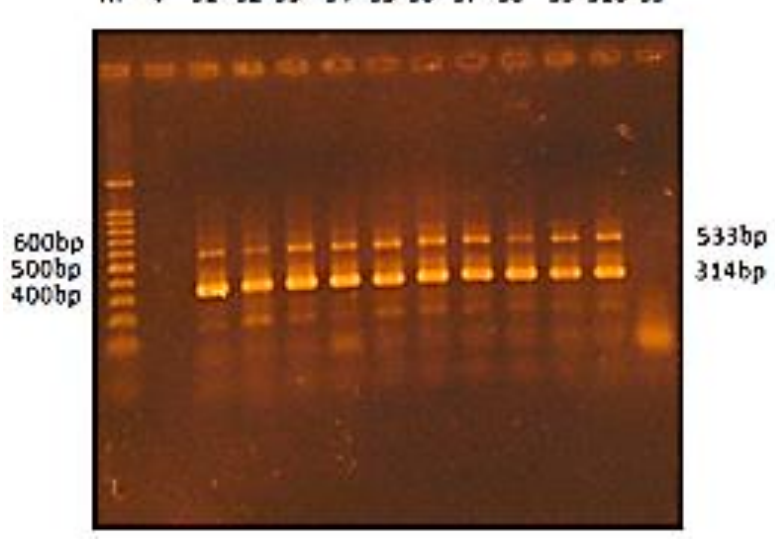

Fig.(4) Ethedium bromide stained agarose gel electrophoresis $1.5 \%$ of amplified PCR products of FemA (314bp) and mecA (533bp) genes of S. aureus isolates. M: 100bp marker, -v: negative control, S3-S12:samples, SS: sensitive isolate.
Many research groups published their primers and probes for detection of mecA efficiently in real time PCR assay. Twenty five published primers sequences were collected and analyzed by in silico PCR online software Table (1).

In silico PCR amplification results for primers verification.

\begin{tabular}{|c|c|c|}
\hline \multirow{2}{*}{\multicolumn{2}{|c|}{ Staphylococci species and strains (Accession number) }} & \multirow{2}{*}{$\begin{array}{c}\text { Amplification results } \\
\text { mecA } \\
\end{array}$} \\
\hline & & \\
\hline 1 & Staphylococcus aureus RF122 (AJ938182.1) & - \\
\hline 2 & Staphylococcus aureus strain Mu50 (BA000017.4) & + \\
\hline 3 & Staphylococcus aureus subsp. aureus 11819-97 (CP003194.1) & + \\
\hline 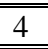 & Staphylococcus aureus subsp. aureus 71193 (CP003045.1) & 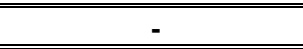 \\
\hline 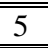 & Staphylococcus aureus subsp. aureus COL (CP000046.1) & + \\
\hline 6 & Staphylococcus aureus subsp. aureus ECT-R 2 (FR714927.1) & - \\
\hline 7 & Staphylococcus aureus subsp. aureus ED133 (CP001996.1) & - \\
\hline 8 & Staphylococcus aureus subsp. aureus ED98 (CP001781.1) & 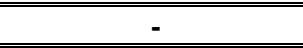 \\
\hline 9 & Staphylococcus aureus subsp. aureus JH1 (CP000736.1) & + \\
\hline 10 & Staphylococcus aureus subsp. aureus JH9 (CP000703.1) & + \\
\hline 11 & Staphylococcus aureus subsp. aureus JKD6159 (CP002114.2) & + \\
\hline 12 & Staphylococcus aureus subsp. aureus M013 (CP003166.1) & + \\
\hline 13 & Staphylococcus aureus subsp. aureus MRSA252 (NC002952.2) & + \\
\hline 14 & Staphylococcus aureus subsp. aureus MSHR1132 (NC016941.1) & + \\
\hline 15 & Staphylococcus aureus subsp. aureus MSSA476 (BX571857.1) & - \\
\hline 16 & Staphylococcus aureus subsp. aureus MW2 (NC003923.1) & + \\
\hline 17 & Staphylococcus aureus subsp. aureus Mu3 (NC009782.1) & + \\
\hline 18 & Staphylococcus aureus subsp. aureus N315 (NC002745.2) & + \\
\hline 19 & Staphylococcus aureus subsp. aureus NCTC 8325 (JN571546.1) & - \\
\hline 20 & Staphylococcus aureus subsp. aureus T0131 (CP002643.1) & + \\
\hline 21 & Staphylococcus aureus subsp. aureus TCH60 (NC017342.1) & + \\
\hline 22 & Staphylococcus aureus subsp. aureus USA300_FPR3757 (CP000255.1) & + \\
\hline 23 & Staphylococcus aureus subsp. aureus USA300_TCH1516 (CP000730.1) & + \\
\hline 24 & Staphylococcus aureus subsp. aureus VC40 (CP003033.1) & - \\
\hline 25 & "Staphylococcus aureus subsp. aureus str. JKD6008 (NC_017341.1) & + \\
\hline 26 & Staphylococcus aureus subsp. aureus str. Newman (NC_009641.1) & - \\
\hline 27 & "Staphylococcus carnosus subsp. carnosus TM300 (AM295250.1) & - \\
\hline 28 & Staphylococcus epidermidis ATCC 12228 (NC004461.1) & - \\
\hline 29 & Staphylococcus epidermidis RP62A (NC004461.1) & - \\
\hline 30 & Staphylococcus haemolyticus JCSC1435 (NC007168.1) & 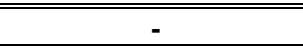 \\
\hline 31 & Staphylococcus lugdunensis HKU09-01 (NC007168.1) & 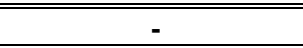 \\
\hline 32 & Staphylococcus lugdunensis N920143 (NC017353.1) & 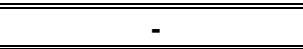 \\
\hline 33 & Staphylococcus pseudintermedius ED99 (NC017568.1) & - \\
\hline 34 & Staphylococcus pseudintermedius HKU10-03 (NC014925.1) & - \\
\hline 35 & "Staphylococcus saprophyticus subsp. Saprophyticus (AP008934.1) & 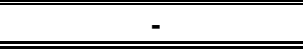 \\
\hline
\end{tabular}


Selection primers that in silico amplify most $S$. aureus isolates increase the chance that these primers will amplify genes in our local isolates.

The six primer pairs amplified in most MRSA strains were analyzed by oligoanalyzer 3.1 software to select the best primer pair Table (2).

Table (2)

The best six primers pairs analyzed by oligoanalyzer 3.1 software.

\begin{tabular}{|c|c|c|c|c|c|c|c|}
\hline primers & Length & $\begin{array}{l}\text { Melting } \\
\text { Temp. }\end{array}$ & $\begin{array}{c}\text { GC } \\
\text { content }\end{array}$ & hairpin & $\begin{array}{c}\text { Maximum } \\
\mathbf{G} \\
\end{array}$ & $\begin{array}{c}\text { Hybridization } \\
\text { Temp. } \\
\end{array}$ & $\begin{array}{c}\text { Mismatch } \\
\text { Tm } \\
\end{array}$ \\
\hline Forward & 22 & $51.8^{\circ} \mathrm{C}$ & $36.4 \%$ & & $\begin{array}{c}-38.91 \\
\mathrm{kcal} / \mathrm{mole}\end{array}$ & $51.8^{\circ} \mathrm{C}$ & $51.8^{\circ} \mathrm{C}$ \\
\hline Reverse & 20 & $55.4^{\circ} \mathrm{C}$ & $55.0 \%$ & $3, \quad T+20$ & $\begin{array}{c}-38.23 \\
\mathrm{kcal} / \mathrm{mole}\end{array}$ & $55.4^{\circ} \mathrm{C}$ & $55.4^{\circ} \mathrm{C}$ \\
\hline Forward & 22 & $54.5^{\circ} \mathrm{C}$ & $40.9 \%$ & 7 structures & $\begin{array}{c}-38.54 \\
\mathrm{kcal} / \mathrm{mole} \\
\mathrm{G}-46.2 \\
\mathrm{kcal} / \mathrm{mole}\end{array}$ & $54.5^{\circ} \mathrm{C}$ & $54.5^{\circ} \mathrm{C}$ \\
\hline Reverse & 26 & $53.9^{\circ} \mathrm{C}$ & $30.8 \%$ & 5 structures & $\begin{array}{c}-46.2 \\
\mathrm{kcal} / \mathrm{mole}\end{array}$ & $53.9^{\circ} \mathrm{C}$ & $53.9^{\circ} \mathrm{C}$ \\
\hline Forward & 21 & $53.9^{\circ} \mathrm{C}$ & $42.9 \%$ & & $\begin{array}{c}-39.22 \\
\mathrm{kcal} / \mathrm{mole}\end{array}$ & $53.8^{\circ} \mathrm{C}$ & $53.9^{\circ} \mathrm{C}$ \\
\hline Reverse & 21 & $53.8^{\circ} \mathrm{C}$ & $42.9 \%$ & 3 structure & $\begin{array}{c}-39.33 \\
\mathrm{kcal} / \mathrm{mole}\end{array}$ & $53.8^{\circ} \mathrm{C}$ & $53.8^{\circ} \mathrm{C}$ \\
\hline Forward & 20 & $52.3^{\circ} \mathrm{C}$ & $45.0 \%$ & One structure & $\begin{array}{c}-37.27 \\
\mathrm{kcal} / \mathrm{mole} \\
-37.75 \\
\mathrm{kcal} / \mathrm{mole}\end{array}$ & $52.3^{\circ} \mathrm{C}$ & $52.3^{\circ} \mathrm{C}$ \\
\hline Reverse & 20 & $51.7^{\circ} \mathrm{C}$ & $40.0 \%$ & 3 structures & $\begin{array}{c}-37.75 \\
\mathrm{kcal} / \mathrm{mole}\end{array}$ & $51.7^{\circ} \mathrm{C}$ & $51.7^{\circ} \mathrm{C}$ \\
\hline Forward & 22 & $52.6^{\circ} \mathrm{C}$ & $36.4 \%$ & One structure & $\begin{array}{c}-41.68 \\
\mathrm{kcal} / \mathrm{mole}\end{array}$ & $52.6^{\circ} \mathrm{C}$ & $52.6^{\circ} \mathrm{C}$ \\
\hline Reverse & 21 & $56.3^{\circ} \mathrm{C}$ & $47.6 \%$ & 3 structures & $\begin{array}{c}-39.91 \\
\mathrm{kcal} / \mathrm{mole}\end{array}$ & $56.3^{\circ} \mathrm{C}$ & $56.3^{\circ} \mathrm{C}$ \\
\hline Forward & 20 & $56.1^{\circ} \mathrm{C}$ & $50.0 \%$ & 6 structures & $\begin{array}{c}-39.68 \\
\mathrm{kcal} / \mathrm{mole} \\
\\
-42.35 \\
\mathrm{kcal} / \mathrm{mole}\end{array}$ & $56.1^{\circ} \mathrm{C}$ & $56.1^{\circ} \mathrm{C}$ \\
\hline Reverse & 24 & $54.8^{\circ} \mathrm{C}$ & $37.5 \%$ & 5 structure & $\begin{array}{c}-42.35 \\
\mathrm{kcal} / \mathrm{mole}\end{array}$ & $54.8^{\circ} \mathrm{C}$ & $54.8^{\circ} \mathrm{C}$ \\
\hline
\end{tabular}

Probes are the most expensive item in real time PCR assay, so in silico prediction of the best probe will reduce the cost for development and evaluation of these assays. Table 3 shows the six probes amplified in most MRSA strains were analyzed by oligoanalyzer 3.1 software to select the best probe. The optimum values selected for parameters testes is according to the guidelines of probe design [10]. 
Table (3)

The best six probes analyzed by oligoanalyzer 3.1 software.

\begin{tabular}{|c||c||c||c||c||c||c||c||}
\hline probe & length & $\begin{array}{c}\text { MeltT } \\
\text { emp }\end{array}$ & $\begin{array}{c}\text { GC } \\
\text { content }\end{array}$ & hairpin & $\begin{array}{c}\text { Maximum } \\
\Delta \mathbf{G}\end{array}$ & $\begin{array}{c}\text { Hybridization } \\
\text { Temp }\end{array}$ & $\begin{array}{c}\text { Probe } \\
\text { sequence }\end{array}$ \\
\hline \hline 1 & 35 & $62.7^{\circ} \mathrm{C}$ & $45.7 \%$ & $\begin{array}{c}\text { One } \\
\text { structure }\end{array}$ & $\begin{array}{c}\mathrm{G}-68.25 \\
\mathrm{kcal} / \mathrm{mole} \\
-68.25 \\
\mathrm{kcal} / \mathrm{mole}\end{array}$ & $62.7^{\circ} \mathrm{C}$ & $\begin{array}{c}\text { GATGGCAAAGATATTC } \\
\text { AACTAACTA }\end{array}$ \\
\hline \hline 2 & 28 & $58.7^{\circ} \mathrm{C}$ & $42.9 \%$ & $\begin{array}{c}\text { One } \\
\text { structure }\end{array}$ & $\begin{array}{c}-48.96 \\
\mathrm{kcal} / \mathrm{mole}\end{array}$ & $58.7^{\circ} \mathrm{C}$ & $\begin{array}{c}\text { CC AGA TTA CAA CTT } \\
\text { CAC CAG GTT CAA CT }\end{array}$ \\
\hline \hline 3 & 24 & $59.8^{\circ} \mathrm{C}$ & $45.8 \%$ & $\begin{array}{c}\text { One } \\
\text { structure }\end{array}$ & $\begin{array}{c}-48.64 \\
\mathrm{kcal} / \mathrm{mole}\end{array}$ & $59.8^{\circ} \mathrm{C}$ & $\begin{array}{c}\text { ACCGAAACAATGTGGA } \\
\text { ATTGGCCA }\end{array}$ \\
\hline \hline 5 & 21 & $57.1^{\circ} \mathrm{C}$ & $47.6 \%$ & $\begin{array}{c}\text { One } \\
\text { structure }\end{array}$ & $\begin{array}{c}-40.98 \\
\mathrm{kcal} / \mathrm{mole}\end{array}$ & $57.1^{\circ} \mathrm{C}$ & $\begin{array}{c}\text { TTGGCCAATACAGGAA } \\
\text { CAGCA }\end{array}$ \\
\hline \hline 6 & 31 & $61.1^{\circ} \mathrm{C}$ & $45.2 \%$ & $\begin{array}{c}\text { One } \\
\text { structure }\end{array}$ & $\begin{array}{c}-58.26 \\
\mathrm{kcal} / \mathrm{mole}\end{array}$ & $61.1^{\circ} \mathrm{C}$ & $\begin{array}{c}\text { CCTTGTTTCATTTTGAG } \\
\text { TTCTGCAGTACCGG }\end{array}$ \\
\hline \hline
\end{tabular}

Real time PCR amplification of in silico selected primers and probe were analyzed by fast 7500 real time PCR Fig.(5). The gene mecA amplified in all MRSA isolates detected by disk diffusion method which indicate the usefulness of in silico analysis before real time PCR assay performance.

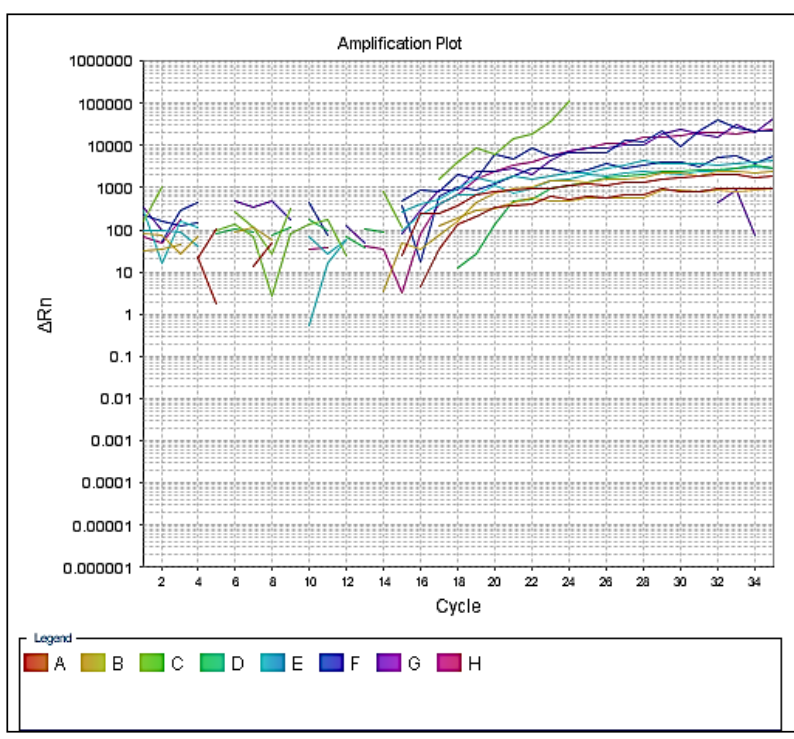

Fig.(5): Real-time amplification curves of mecA gene.

To confirm real time amplification of mecA gene result, PCR products were analyzed by agarose gel electrophoresis stained with ethidium bromide Fig.(6).

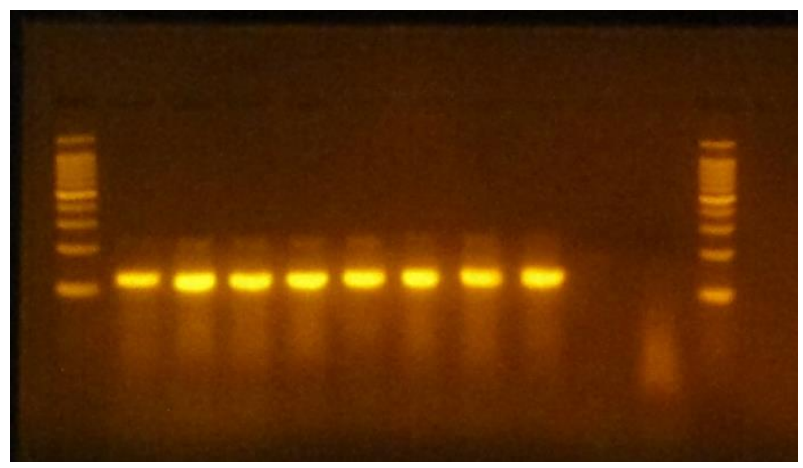

Fig.(6): Agarose gel electrophoresis (1.5\%) of mecA (124bp) gene products (amplified in real time PCR).

In silico PCR approach in this work act as prescreen for custom-designed primers. This will reduce the cost for purchasing unwanted primer pairs as well as decrease the time spent on trial and error of the primer selection. The in silico primers and probe showed a good correlation with actual real-time PCR detection mecA genes towards identification of local methicillin-resistant $S$. aureus (MRSA).

\section{References}

[1] Haddadin A, Fappiano S, and Lipsett P, "Methicillin resistant Staphylococcus aureus (MRSA) in the intensive care unit". Postgrad Med J.; 78, 385-392, 2002.

[2] Zurita J, Mejía C, and GuzmánBlanco M, "Diagnosis and susceptibility testing of methicillin-resistant Staphylococcus aureus in Latin America". Braz J Infect Dis; 14, S97-S106, 2010. 
[3] Unal S, Hoskins J, Flokowitsch J, and WU C, "Detection of Methicillin-Resistant Staphylococci by Using the Polymerase Chain reaction". Journal of Clinical Microbiology; 30, 1685-1691, 1992.

[4] Kuźma K, Malinowski E, Lassa H, and Kłossowska A, "Specific detection of Staphylococcus aureus by PCR in intramammary infection". Bull. Vet. Inst. Pulawy; 47, 183-190, 2003.

[5] Awad S, Alshami I and Alharbi E, "Evaluation of a duplex real-time PCR assay to detect MRSA from broth culture, human sera seeded with MRSA and from patient's serum". Bioinformation; 9, 896900, 2013.

[6] Grisold A, Leitner E, Mühlbauer G, Marth $\mathrm{E}$, and Kessler H, "Detection of Methicillin-Resistant Staphylococcus aureus and Simultaneous Confirmation by Automated Nucleic Acid Extraction and Real-Time PCR". J Clin. Microbiol; 2002, 40, 2392-2397, 2002.

[7] Costa AM, Kay I, and Palladino S, "Rapid detection of mecA and nuc genes in staphylococci by real-time multiplex polymerase chain reaction". Diagn. Microbiol; Infect. Dis. 51: 13-7, 2005.

[8] Suhaili Z, Johari S, Sajili M, Yahya A, Zakaria Z, Desa M, and Ali A, "In silico PCR Verification and Simplex Real-Time PCR Detection of Methicillin-Resistant Staphylococcus aureus (MRSA) from East Coast Malaysian Clinical Isolates". Walailak J Sci \& Tech; 10, 1-12, 2013.

[9] Sowmya N, Thakur M, and Manonmani $\mathrm{H}$, "Rapid and simple DNA extraction method for the detection of enterotoxigenic Staphylococcus aureus directly from food samples: comparison of PCR and LAMP methods". Journal of Applied Microbiology; 113, 106-113, 2012.

[10] Bustin S, Benes V, Garson J, Hellemans J, Huggett J, Kubista M, Mueller R, Nolan T, Pfaffl M, Shipley G, Vandesompele J, and Wittwer C, "The MIQE Guidelines: Minimum Information for Publication of Quantitative Real-Time PCR Experiments". Clinical Chemistry; 55, 4-16, 2009.

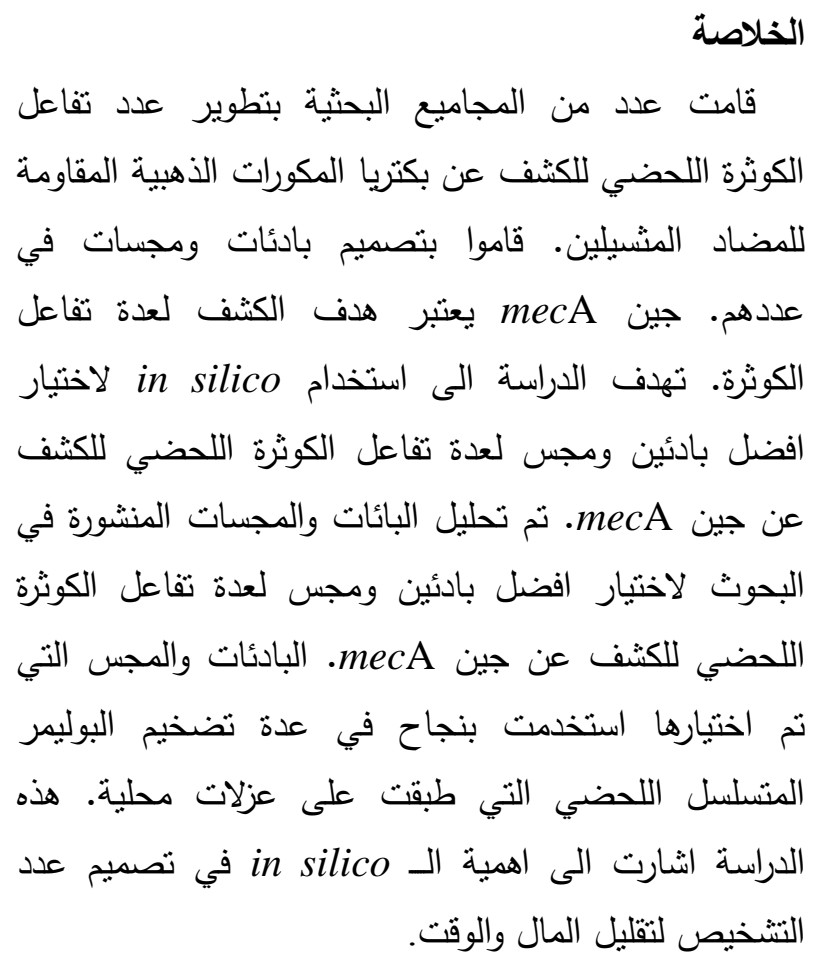

